\title{
Vom Überleben des Menschenrechts auf Privatheit in der digitalen Revolution
}

Vor zehn Jahren begann in Folge der Ereignisse von 9/11 eine Präventionspolitik, die trotz Überwindung der ersten Schockstarre weiter darauf abzielt, hochrangige Rechtsgüter wie das Recht auf Privatheit, die Unverletzlichkeit der Wohnung oder das Telekommunikationsgeheimnis massiv einzuschränken. Mit diesem gravierenden Problem befasst sich Gerhart Baum und fordert den Gesetzgeber auf, sich für den "unverzichtbaren Schutz der Privatheit" in gefährdeten Bereichen (Vorratsdatenspeicherung, Elena usw.) auch gegen die "German Angst" und den verbreiteten Sicherheitswahn vieler Bürgerinnen und Bürger einzusetzen. Die Unterzeichnerin zeigt die Transformation der Privatheit aus alten in neue, vernetzte Räume. Sie verweist auf die wechselseitige Abhängigkeit der realen und virtuellen Räume und betont, dass eine intakte Umwelt notwendige Voraussetzung der Privatheit ist, worauf auch die Rechtsprechung des Europäischen Gerichtshofes (EGMR) hindeute. Auch Rudolf Streinz befasst sich mit dieser Rechtsprechung des EGMR und zwar im Verhältnis zur Rechtsprechung des EuGH. Die "Nagelprobe" kann nach Ansicht des Autors die materielle Prüfung der EU-Richtlinie zur Vorratsdatenspeicherung sein, wenn deren Umsetzungsprobleme und unzureichende Änderungen, wie sie im Folgenden auch von Thomas Petri erörtert werden, als entsprechende Vorlagen zu den beiden Gerichten gelangen. Petri setzt sich detailliert mit diesen Fragen im Kompetenzbereich der EU und auf der Folie der europäischen Grundrechte auseinander, über die im Besonderen der EGMR zu wachen hat.

Bernd Holznagel und Thorsten Ricke analysieren die noch wenig erforschten Möglichkeiten und Grenzen der Gewährleistung von Netzneutralität im Zusammenhang mit dem deutschen Telekommunikations- und Medienrecht. Die Autoren stellen fest, dass die technische Funktionsweise des Internets vor grundlegenden Änderungen steht. Phillip W. Brunst zeigt aktuelle technische und rechtliche Ermittlungsinstrumente und Entwicklungen, die zu einer Erosion der Anonymität im Internet führen, wobei er die Vorratsdatenspeicherung als gegenwärtig größte Gefahr einordnet. Der Autor verweist aber auch auf die Verantwortung jedes Einzelnen für selbst preisgegebene Daten. Auch Jerry Kang hat eine solche Eigenverantwortung vor Augen, wenn er das Problem der freiwilligen Selbstüberwachung (self-surveillance) anspricht; für eine effektive Ausübung des Selbstdatenschutzes führt der Autor das Modell des „Personal Data Guardian“ ein.

Der Konzerndatenschutz soll im Gegensatz zum Beschäftigtendatenschutz auf EUEbene gesetzlich verankert werden. Hans-Hermann Schild und Marie-Theres Tinnefeld erörtern EU-relevante Fragen eines Konzernprivilegs unter besonderer Berücksichtigung von SAP-Anwendungen.

Die sich anschließenden Beiträge geben sodann einen Überblick über aktuelle datenschutzrechtliche Entwicklungen im Ausland. Burkhard Schafer untersucht die Weiterentwicklung von Privacy in "post-Labour Britain“. Christina Hermida del Llano unternimmt eine Darstellung des Datenschutzes in Spanien im Kontext der europäischen Datenschutzentwicklung. Von besonderem Interesse ist die Untersuchung der chinesischen Autorin Ge Jiang, die differenziert die Versuche Chinas in Richtung einer Datenschutzgesetzgebung darlegt.

Last but not least setzt sich Stephan Doesinger kritisch mit der Idee eines so genannten "Medienführerscheins" auseinander - aus Sicht des Autors eher eine "Geisterfahrt" denn ein Weg hin zu mehr Eigenverantwortung und Privatheitsschutz.

Marie-Theres Tinnefeld, Benedikt Buchner 